\title{
Submitted: 1 Sonographic assessment of the anatomy and common Accepted: pathologies of clinically important bursae
} 25.07.2019

Published: 30.09.2019

Keywords
high-resolution
ultrasonography,
bursa,
anatomy,
inflammation

\author{
Slavcho Ivanoski ${ }^{1}$, Violeta Vasilevska Nikodinovska² \\ ${ }^{1}$ Special Hospital for Orthopedic Surgery and Traumatology "St. Erasmus", Ohrid, \\ Macedonia \\ ${ }^{2}$ University Surgical Clinic "St. Naum Ohridski" Skopje, University "Ss. Cyril \\ and Methodius", Skopje, Macedonia \\ Correspondence: Slavcho Ivanoski, Bul. Mak. Prosvetiteli 9, Ohrid, R. Macedonia; \\ tel.: +38970815573, e-mail: slavcoivanoski@gmail.com
}

DOI: $10.15557 / J o U .2019 .0032$

\begin{abstract}
High-resolution ultrasonography has many advantages in the imaging of the musculoskeletal system, when compared to other imaging methods, particularly in superficial, easily accessible parts of the body. It is a perfect diagnostic tool for visualizing the most common pathologies of the musculoskeletal system, including the bursae. Inflammation of bursae is frequent, and it can mimic other diseases of the musculoskeletal system. Therefore, knowledge of normal ultrasound anatomy of the bursae, their exact location in the human body, and the sonographic signs of their most common pathologies is essential for establishing a quick and accurate diagnosis by ultrasound. Common conditions affecting bursae, leading to bursitis, include acute trauma, overuse syndromes, degenerative diseases, inflammatory conditions (rheumatoid arthritis, psoriatic arthritis, gout etc.), infections such as tuberculosis, synovial tumors and tumor-like conditions (pigmented villonodular synovitis, osteochondromatosis), and many more. This review article presents and explains ultrasound examples of the most frequent pathological conditions affecting bursae. Images include normal and pathological conditions of bursae around the shoulder joint, elbow, hip, knee, and ankle joint.
\end{abstract}

\section{Introduction}

Bursae are defined as sac-like structures, normally filled with a small amount of fluid, communicating or not communicating with the adjacent joints, whose function is to reduce the friction between two anatomical structures, tendons or muscles and bones or the skin ${ }^{(1)}$. Bursae can be either constant or adventitial. Constant bursae are formed during embryological development, lined with synovial cells, containing small amounts of synovial fluid, always appearing in the same, predictable and defined spots. Adventitial bursae are formed later during life, at sites of chronic friction or pressure, and are not lined with any synovial layer. There are about 160 different constant bursae in the human body ${ }^{(2)}$.

Bursitis refers to the inflammation of a bursa, and it is characterized by synovial thickening, increased amount of fluid within the bursa, localized pain near the inflammation, and adjacent soft tissue swelling ${ }^{(3)}$. Bursitis occurs in many pathological conditions. They include acute traumatic injury of the bursa; repetitive microtrauma; degenerative diseases; inflammatory conditions affecting synovia, most commonly rheumatoid arthritis and gout; various infections; synovial tumors or pseudotumors like pigmented villonodular synovitis, synovial osteochondromatosis, and many other pathologies ${ }^{(4)}$.

Ultrasonography can be a valuable tool for the detection and evaluation of bursitis. Its specificity and sensitivity in the assessment of bursal pathology, especially in superficially localized bursae, is comparable to MRI ${ }^{(5)}$. Normal bursae are usually barely or not visible at all using ultrasound (Fig. 1). A major ultrasound sign of bursitis is the enlargement of a bursa, with an increased amount of fluid within it, which can be anechoic in some 
cases. In other cases, increased echogenicity is apparent due to debris, blood in acute trauma, or puss - if infection appears. Other signs include thickening of the hyperechoic synovial wall, which can be either uniform or irregular. The latter sign is more often present in cases of chronic bursitis. Hyperechoic edema of surrounding soft tissue or lymphedema with increased vascularity on Doppler is a common sonographic sign of bursitis (Fig. 2).

Recent advances in ultrasound imaging could further improve the detection of bursal pathology, and delineate

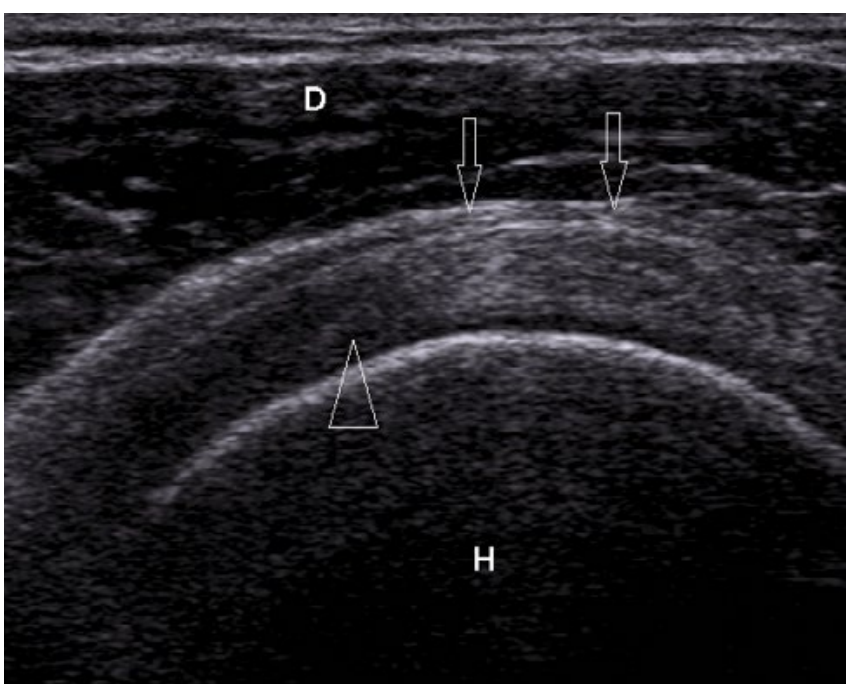

Fig. 1. Normal subacromial-subdeltoid bursa. There is a minimal amount of anechoic fluid within the bursa (arrows), located between the deltoid muscle (D) and the supraspinatus muscle tendon (arrowhead). H-humeral head
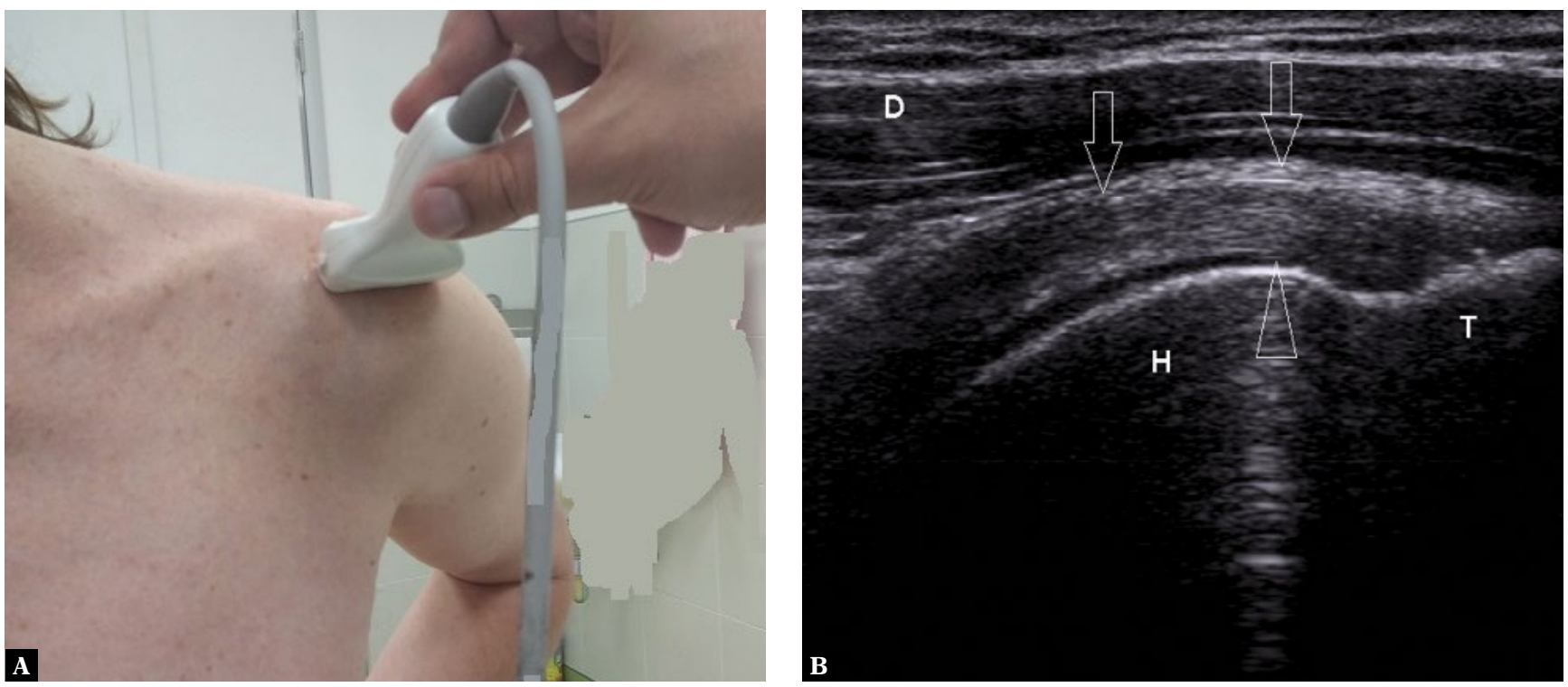

Fig. 3. A. Evaluation of the subacromial-subdeltoid bursa. Positioning of the patient and the transducer. B. Normal subacromial-subdeltoid bursa in the longitudinal plane (arrows). Arrowhead-supraspinatus tendon, H-humeral head, T-tuberculum majus, D-deltoid muscle very small fluid collections, undetectable with conventional ultrasound methods. Several techniques of ultrasound elastography, a qualitative or quantitative method that uses mechanical stress applied to the tissue, and causes changes dependent on elastic tissue properties, show promising results for bursal disease diagnosis. Cases of retrocalcaneal, subacromial-subdeltoid or deep infrapatellar bursae can be detected as small, soft areas, compared to nearby tendons presenting as stiff zones using ultrasound elastography ${ }^{(6)}$.

The purpose of this review article is to demonstrate the anatomical localization of clinically most important bursae

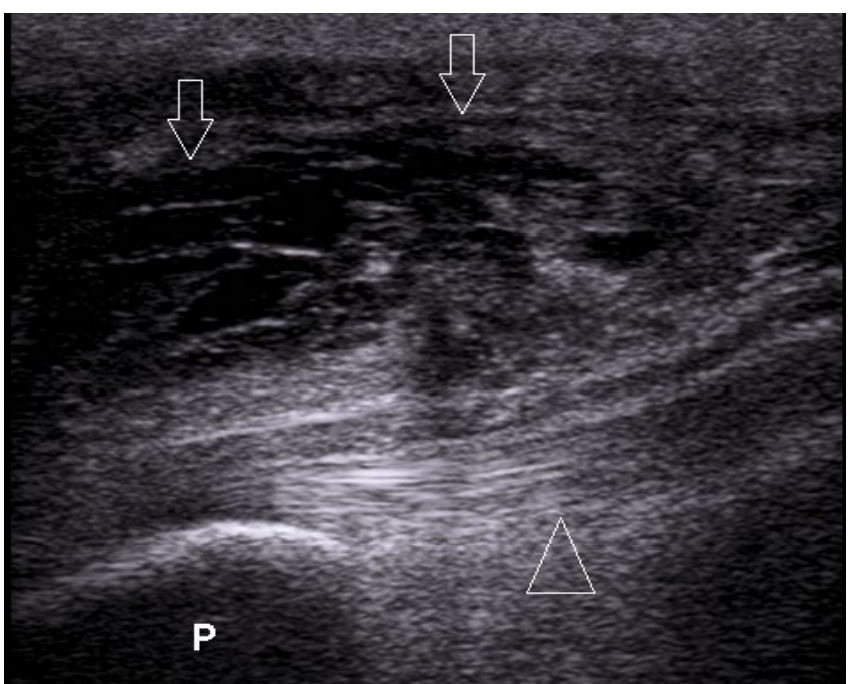

Fig. 2. Bursitis. Prepatellar bursa is filled with fluid, debris, and irregular synovia (arrows). Hyperechoic edema of surrounding soft tissue can be noted. Inflammation of the superficial part of the patellar ligament is also visible (arrowhead). P-patella 
of the upper and lower extremity using high-resolution ultrasonography, and to explain the ultrasound diagnosis of the most frequent and clinically most important pathologies of bursae.

\section{Imaging findings}

\section{Shoulder}

Subacromial-subdeltoid bursa is clinically the most important bursa of the shoulder. However, there are also several other bursae around the shoulder joint: coracobrachial bursa, subcoracoid bursae, and subscapularis subtendinous bursa ${ }^{(7)}$.

Subacromial-subdeltoid bursa is the largest bursa of the human body, but the two walls of the bursa are normally only up to $2 \mathrm{~mm}$ apart $^{(8)}$. It is composed of subacromial and subdeltoid portions, which in the majority of people communicate by a connective tissue band ${ }^{(9)}$. Normal subacromial-subdeltoid bursa is localized between the rotator cuff tendons and the deltoid muscle, and the rotator cuff tendons and the acromion.

For the best representation of the bursa by ultrasound, the patient's hand should be resting on the iliac wing, while the arm should be positioned posteriorly. The transducer should follow the long axis of the supraspinatus tendon (Fig. 3A). The bursa is normally visible on the ultrasound scan as a thin, linear, anechoic structure between hyperechoic peribursal fat, but the synovia is not apparent on ultrasound $^{(8)}$ (Fig. 3B).

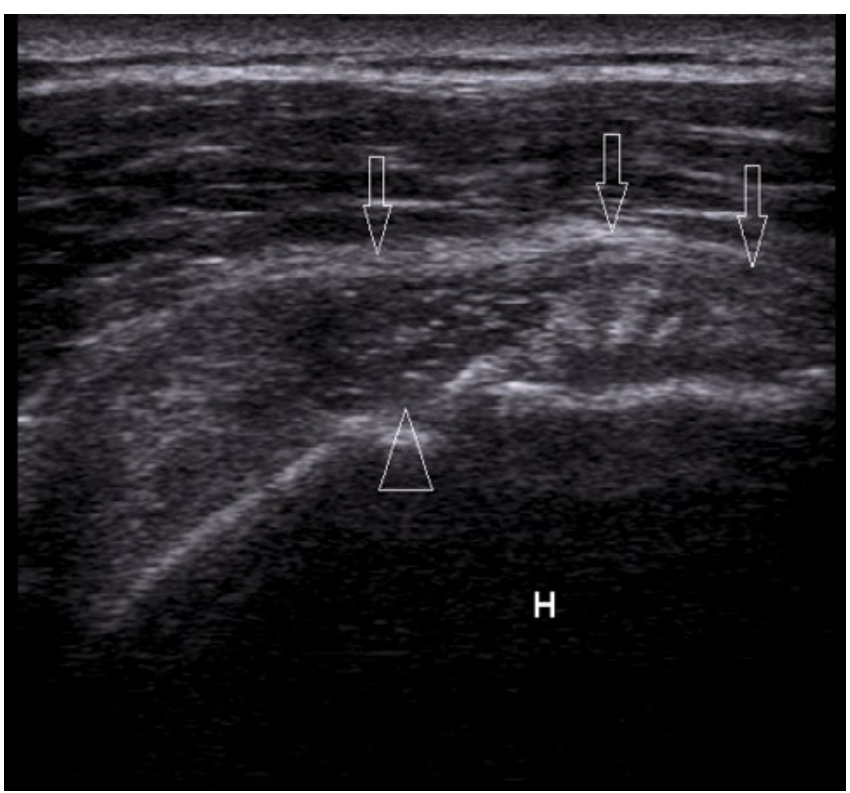

Fig. 4. Communicating subacromial-subdeltoid bursitis. Communication (arrowhead) between the glenohumeral joint and the bursa (arrows) is visible through a total tear of the supraspinatus tendon. The bursa is filled with a moderate amount of hyperechoic fluid. H-humeral head
One may distinguish between communicating and noncommunicating subacromial-subdeltoid bursitis ${ }^{(10)}$. The most common type of subacromial-subdeltoid bursitis is the communicating variant, when the bursa interconnects with the glenohumeral joint. It occurs in cases involving a total rotator cuff tear, either traumatic or degenerative in nature (Fig. 4). Non-communicating subacromial-subdeltoid bursitis could be a result of shoulder overuse, direct traumatic blow to the bursa, osteoarthritis, rheumatoid arthritis, etc. (Fig. 5).

\section{Elbow}

Two clinically important bursae are localized near the elbow. Bicipitoradial bursa, around the distal biceps tendon, and superficial olecranon bursa. Olecranon bursa is the most superficial bursa of the human body. It is positioned between the ulnar olecranon and the skin. Olecranon bursa is normally not visible on ultrasound.

To achieve the best sonographic evaluation of the bursa, the patient's hand and elbow should be in 90 degrees flexion, with the palmar side of the hand facing towards the table. The transducer should be positioned in the longitudinal or transverse plane, over the olecranon of the ulna which is used as a bony landmark (Fig. 6A). Olecranon bursitis often occurs in persons with more pronounced, palpable olecranon (Fig. 6B and C). Common etiology includes a traumatic event or infection; even a minor, sometimes neglected trauma or repetitive microtrauma can lead to olecranon bursitis (also known as student's elbow, miner's elbow) ${ }^{(11)}$. However, septic olecranon bursitis is not uncommon either,

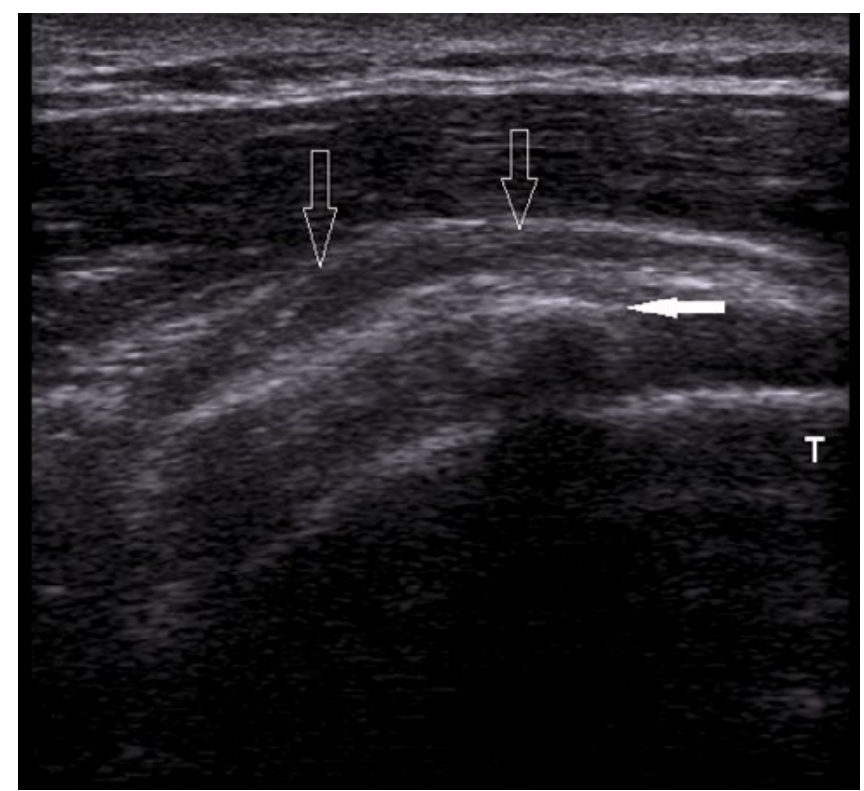

Fig. 5. Non-communicating subacromial-subdeltoid bursitis. An increased amount of fluid in the bursa (arrows) in a patient with calcifying supraspinatus tendinopathy (filled arrow). T-tuberculum majus 
due to its superficial localization and poor vascularity, particularly in immunocompromised patients ${ }^{(12)}$, occurring in about a third of patients with bursal inflammation ${ }^{(11)}$. Olecranon bursa is most commonly affected by inflammation of all the constant bursae ${ }^{(12)}$.

\section{Hip}

There are three bursae around the trochanter major of the femur. Trochanteric bursa, which is the largest of the three, covers the lateral insertion of the medial gluteus muscle and posterior trochanteric facet. Subgluteus medius bursa is located between the tendon of gluteus medius and the lateral facet. Subgluteus minimus bursa can be found between the gluteus minimus tendon and the anterior facet of the femur ${ }^{(13)}$. It is proposed that pain of the lateral hip, known as greater trochanteric pain syndrome, is in most cases a result of pathology involving gluteal tendons or the iliotibial band. Fluid within bursae is secondary to these conditions, the primary trochanteric bursitis being very rare ${ }^{(14)}$.

Iliopsoas bursa is the largest bursa around the hip. For the assessment of the iliopsoas bursa pathology, the patient should be resting supine with $180^{\circ}$ hip extension. The transducer should be positioned in the longitudinal plane, over
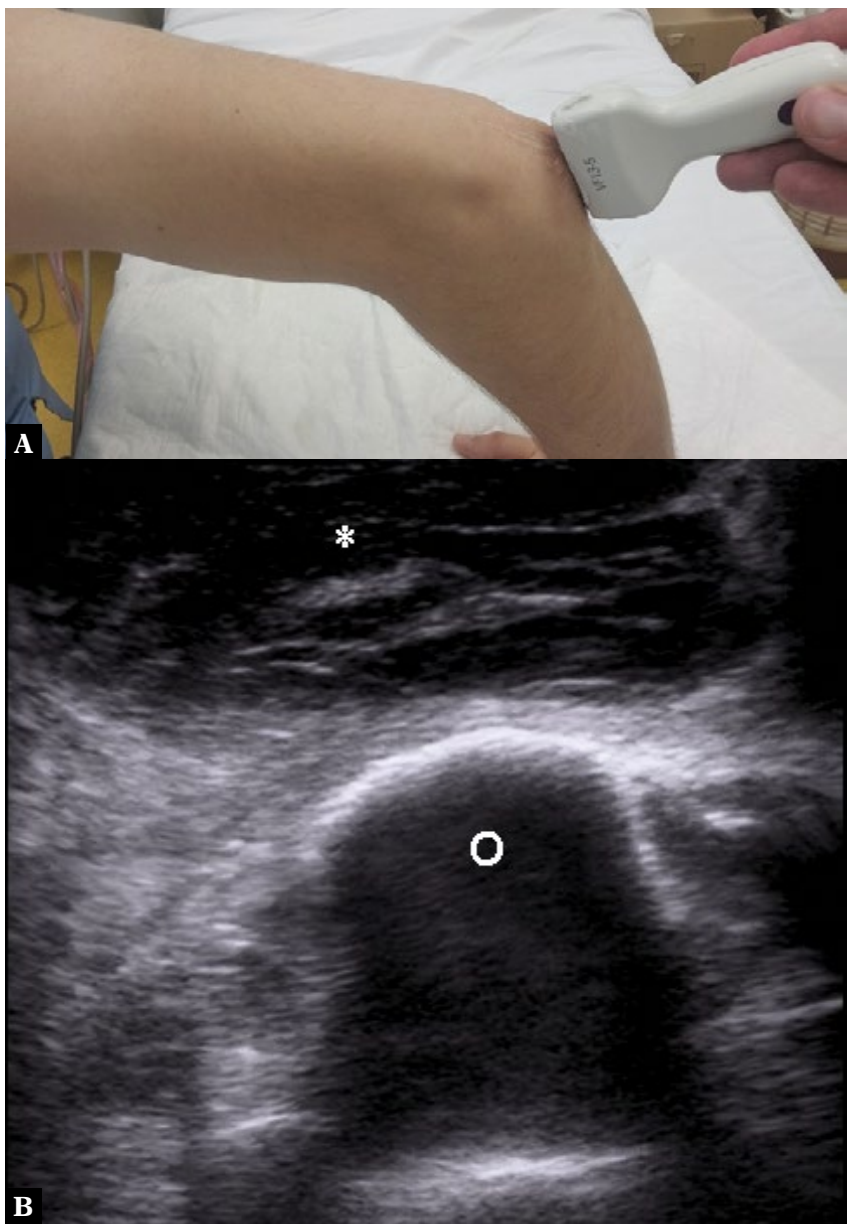

the femoral head and femoral neck which are used as bony landmarks (Fig. 7A). The bursa should be seen between the musculotendinous part of iliopsoas muscle and the anterior capsule of the hip ${ }^{(15)}$ (Fig. 7B). Iliopsoas bursa communicates with the hip joint in $15 \%$ of adults ${ }^{(16)}$, and an enlarged bursa can be a consequence of hip pathology: osteoarthritis, inflammatory disease etc. ${ }^{(17)}$, or primary bursitis.

\section{Knee}

There are several bursae around the knee. They include prepatellar, deep and superficial infrapatellar bursae, and suprapatellar bursa anteriorly to the knee; Baker's cyst in posterior knee compartment; medial collateral ligament bursa (Fig. 8), semimembranosus-medial collateral ligament bursa, and pes anserine bursa medially; lateral collateral ligament bursa, and iliotibial bursa laterally to the knee $^{(5)}$.

During the embryological development, the suprapatellar bursa is divided from the knee joint with a thin septum or plica, but later the plica disappears, and it normally communicates with the knee joint in over $80 \%$ of adult individuals $^{(18)}$. Suprapatellar bursa is situated between

Fig. 6. A. Evaluation of the olecranon bursa. Positioning of the patient and the transducer. B. Superficial olecranon bursitis in the transverse plane (*). O-olecranon. C. Superficial olecranon bursitis in longitudinal plane (*). O-olecranon

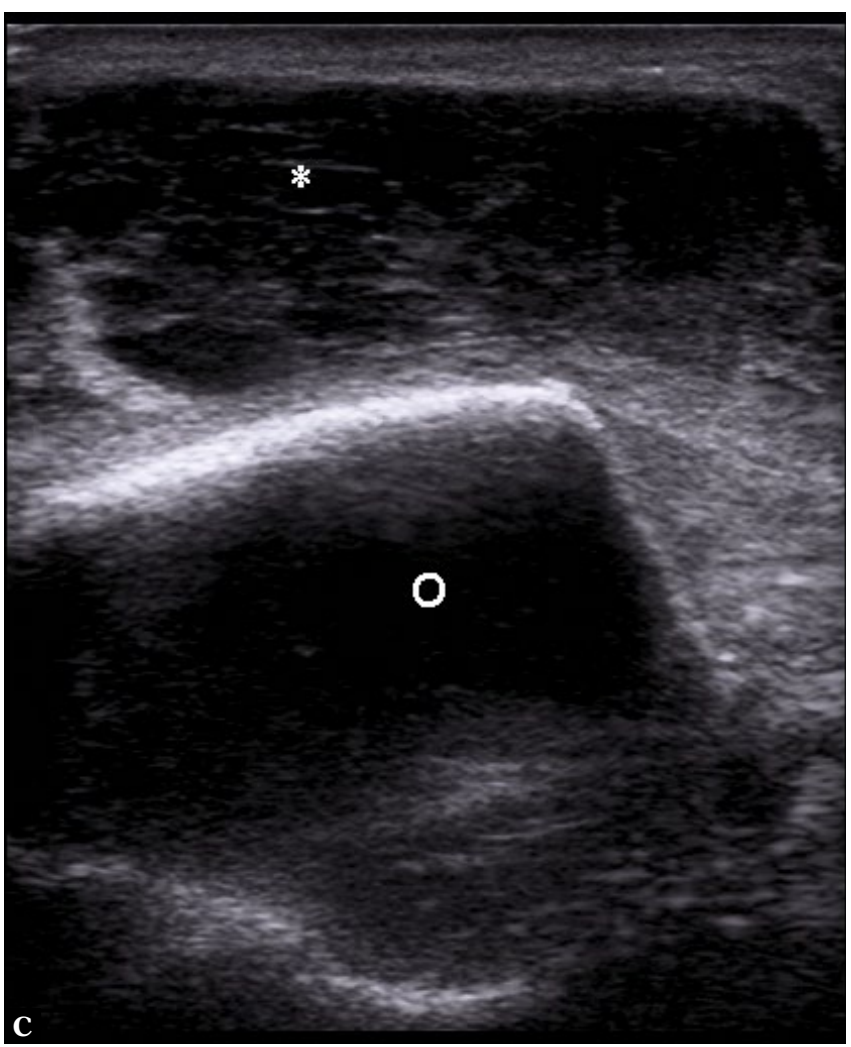




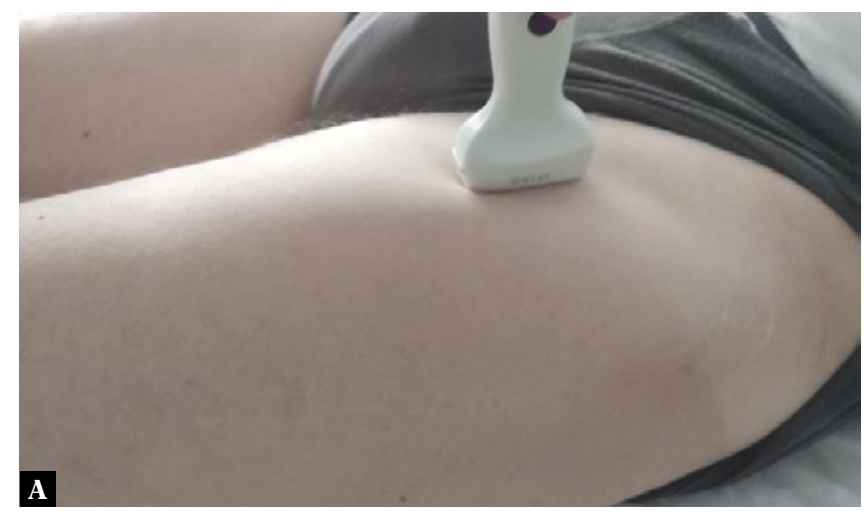

Fig. 7. A. Evaluation of the iliopsoas bursa. Positioning of the patient and the transducer. B. Iliopsoas bursitis in the longitudinal plane. Enlarged, fluid-filled iliopsoas bursa (arrow) is visible deep to the ileopsoas tendon (arrowhead). An increased amount of fluid in the anterior hip recess (*)

the intercondillar fossa of the femur and the quadriceps tendon.

For the sonographic evaluation of the suprapatellar bursa, the patient should rest in the supine position with 25-30 degrees knee flexion. The transducer should be positioned in the longitudinal plane, proximal to the upper pole of the patella used as bony landmark (Fig. 9). Suprapatellar bursa is enlarged in the majority of pathologic conditions affecting the knee and producing excessive fluid within the joint. Even small amounts of fluid in the suprapatellar bursa can be visualized by high-resolution ultrasound, which is comparable to MRI in the detection of fluid and synovitis both in the suprapatellar recess and the Baker's cyst ${ }^{(19,20)}$. The quantity ${ }^{(21)}$, but also the quality of

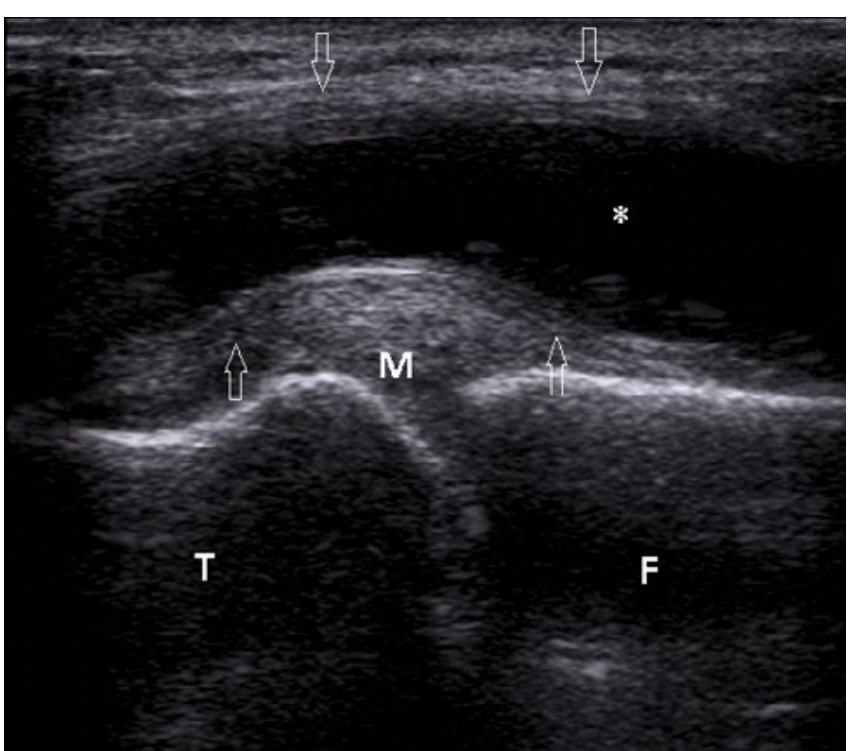

Fig. 8. Medial collateral ligament bursitis. There is a large amount of anechoic fluid filling the bursa (*) between the superficial and deep layers of the medial collateral ligament. M-medial meniscus, T-tibia, F-femur

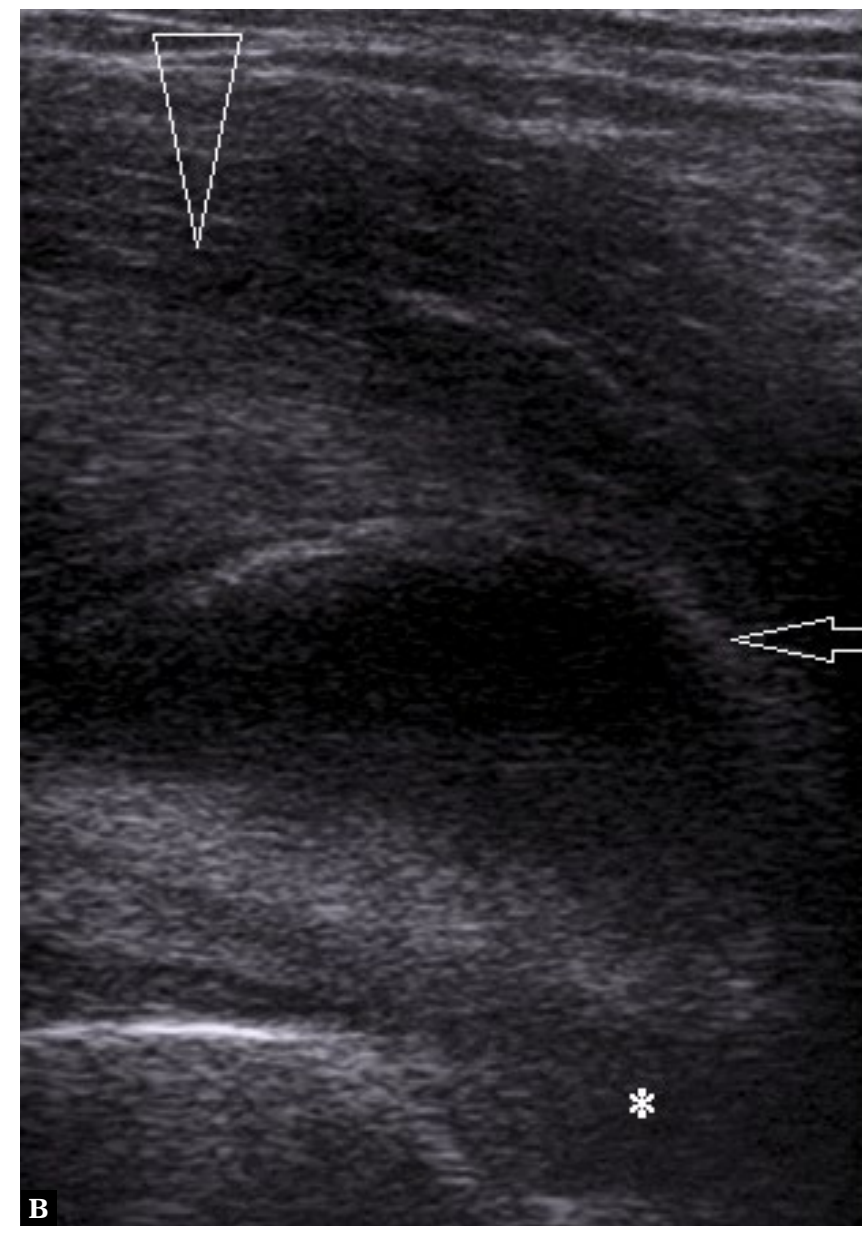

the effusion within the suprapatellar recess or the Baker's cyst can be adequately assessed by ultrasonography ${ }^{(22)}$. The fluid within the recess can be anechoic or hypoechoic (Fig. 10), hyperechoic, containing blood in cases of severe trauma or severe arthritis. Sometimes fat fluid level can be visible within the recess as a sign of intraarticular fracture $^{(22)}$ (Fig. 11).

Baker's cyst is the normal gastrocnemius-semimembranosus bursa, in some adults communicating with the posterior part of the knee capsule through a thin neck ${ }^{(23)}$ (Fig. 12A). Baker's cyst is located between the semimembranosus and medial head of gastrocnemius tendons. To evaluate its presence and pathology by ultrasound, the patient should lie prone with a fully extended knee. The transducer should be placed in the longitudinal or transverse plane, in the medial half of the popliteal fossa, centered between the semimembranosus and medial gastrocnemius tendons which can serve as anatomic landmarks (Fig. 12 B).

Its presence is often related to knee effusion, and it occurs in about $40 \%$ of patients with mass lying posterior to the knee ${ }^{(24)}$. The cyst enlarges frequently in cases of knee overuse, osteoarthritis of the knee, arthritis, pigmented villonodular synovitis, synovial osteochondromatosis 


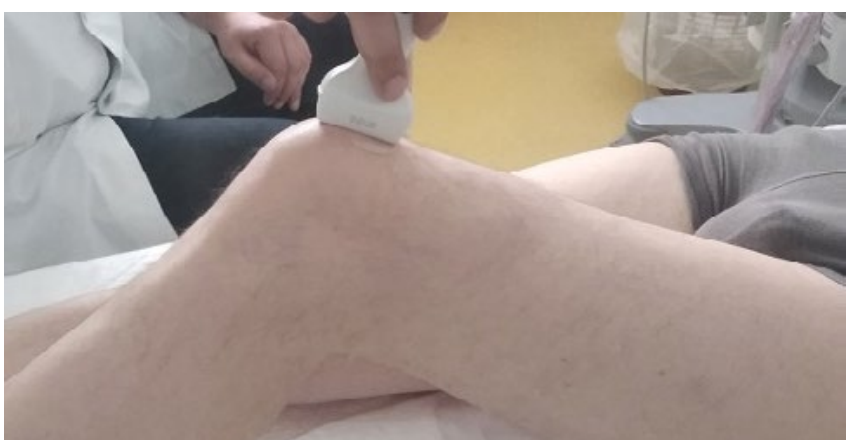

Fig. 9. Evaluation of the suprapatellar bursa. Positioning of the patient and the transducer

(Fig. 12C) etc. Rupture of a Baker's cyst with synovial fluid leakage in the calf is the most common and very painful complication. In case of Baker's cyst rupture, there is a free soft-tissue fluid around the cyst visible on ultrasound.

Prepatellar bursa is normally a very thin structure, filled with a minimal amount of fluid, not visible on ultrasound. It is localized between the patellar surface and the proximal part of the patellar ligament and the skin.

For the sonographic assessment of prepatellar bursitis, the patient should rest supine, with the knee in partial (25-30 degrees) flexion. The transducer should be placed in the longitudinal position, over the inferior patellar pole which is used as a bony landmark (Fig. 13). Prepatellar bursitis, also known as housemaid's knee is a common painful condition, easily visible sonographically (Fig. 14). In the

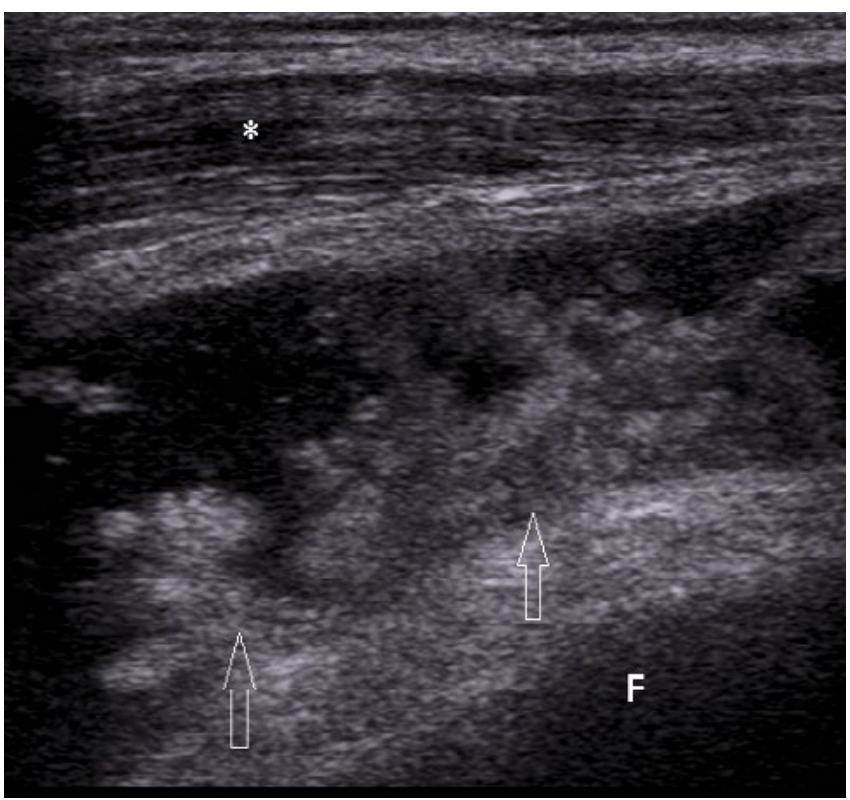

Fig. 10. Pigmented villonodular synovitis of the knee. The suprapatellar bursa is filled with hypoechoic fluid; irregular synovial hypertrophy (arrows). F-femur, *-quadriceps tendon majority of cases, it is a result of chronic overuse. Less commonly, it develops as a consequence of direct traumatic blow $^{(25)}$. Professions involving prolonged kneeling such as floor layers, carpenters, auto mechanics, housemaids and others are especially prone to prepatellar bursitis.

Superficial infrapatellar bursa is normally situated between the skin and the distal part of the patellar ligament. For the best representation of the superficial as well as deep infrapatellar bursa, the patient should lie supine, with a partially flexed (30 degrees) knee. The transducer should be in the longitudinal plane, following the fibrillar structure of the patellar ligament, just above the tibial tuberosity which could be used as bony landmark (Fig. 15). Superficial infrapatellar bursitis is also known as clergyman's knee. In the majority of cases, it results from an acute traumatic injury (direct blow to the bursa) or chronic overuse ${ }^{(26)}$ (Fig. 16).

Deep infrapatellar bursa is located between the inferior part of the patellar ligament and the anterior tibial surface (Fig. 15). Normal bursa in some situations can be visualized by ultrasound as a very small collection of anechoic fluid ${ }^{(27)}$ posteriorly to the patellar ligament (Fig. 17). The etiology of the deep infrapatellar bursitis includes excessive sports activity, especially long-distance running and jumping (Fig. 18).

\section{Ankle}

There are two clinically important bursae near the Achilles tendon $^{(28)}$. The deeper, retrocalcaneal bursa lies between the distal anterior part of the Achilles tendon and the

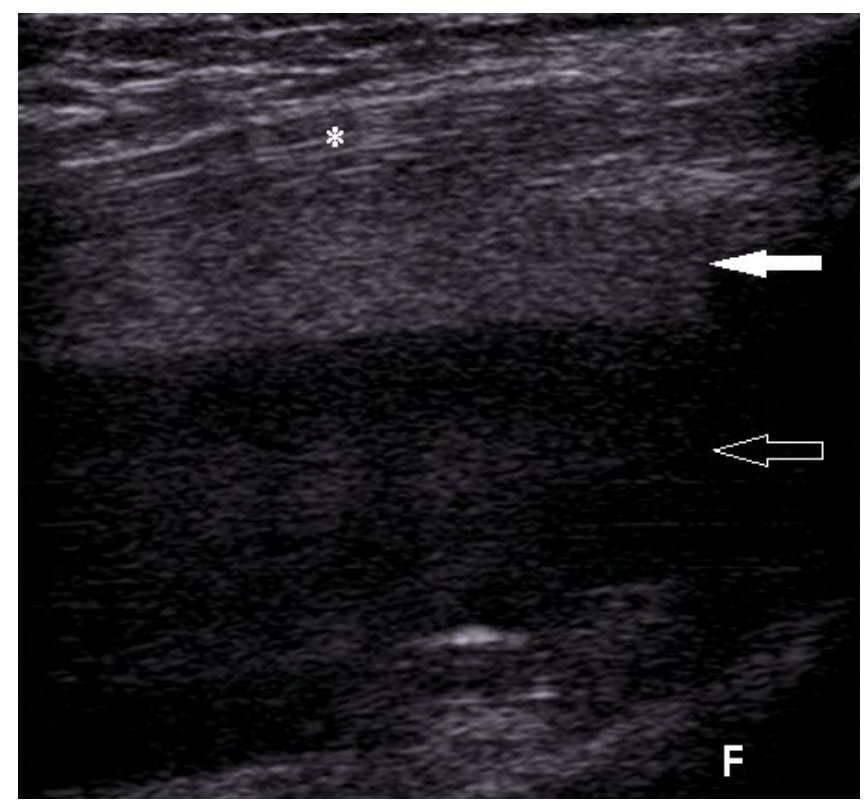

Fig. 11. Lipohemarthrosis. A large amount of hyperechoic fluid (arrow) with fat-fluid level (filled arrow) can be visualized within the recess in a patient with plateau tibial fracture. *-quadriceps tendon, F-Femur 


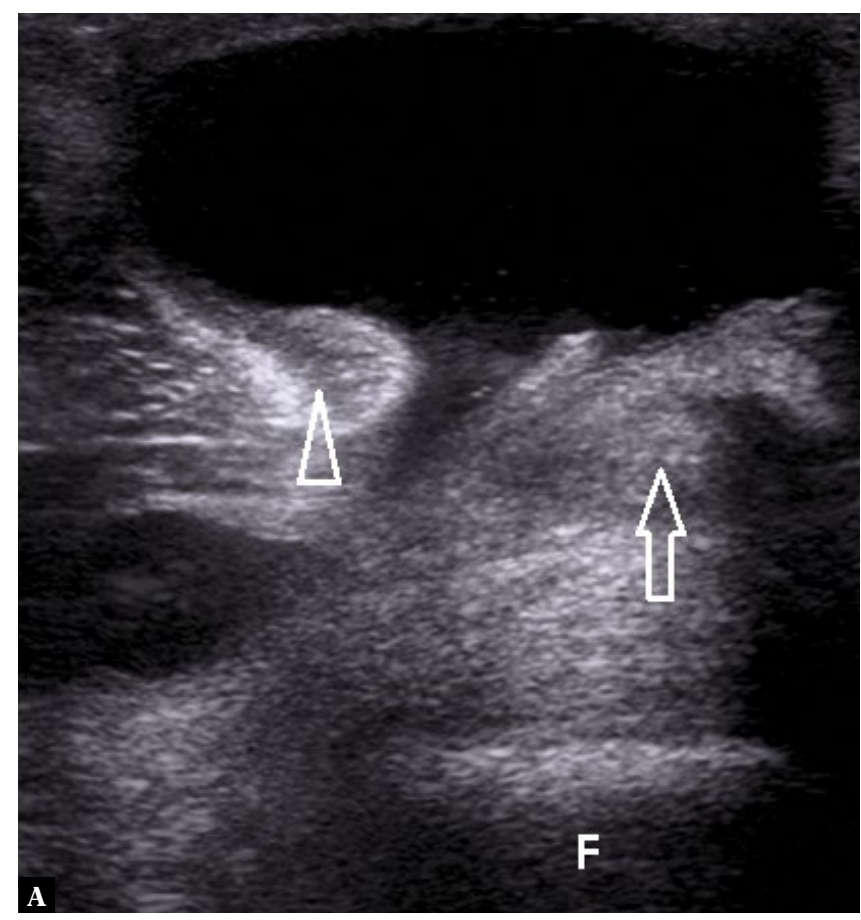

Fig. 12. A. Baker's cyst in the transverse plane, localized between the semimembranosus tendon (arrow) and the medial head of the gastrocnemius muscle (arrowhead). The cyst is communicating with the knee joint through a thin neck. F-Femur. B. Evaluation of Baker's cyst. Positioning of the patient and the transducer. C. Baker's cyst in the longitudinal plane. Several ossified bodies (arrows) can be seen within the cyst due to secondary osteochondromatosis

posterosuperior part of the calcaneus. Normal retrocalcaneal bursa, which is a constant bursa, can be visible on ultrasound in almost $25 \%$ of healthy individuals ${ }^{(27)}$. The superficial, or also called "Achilles", bursa is situated between subcutaneous fat tissue and the distal Achilles tendon or the calcaneal surface.

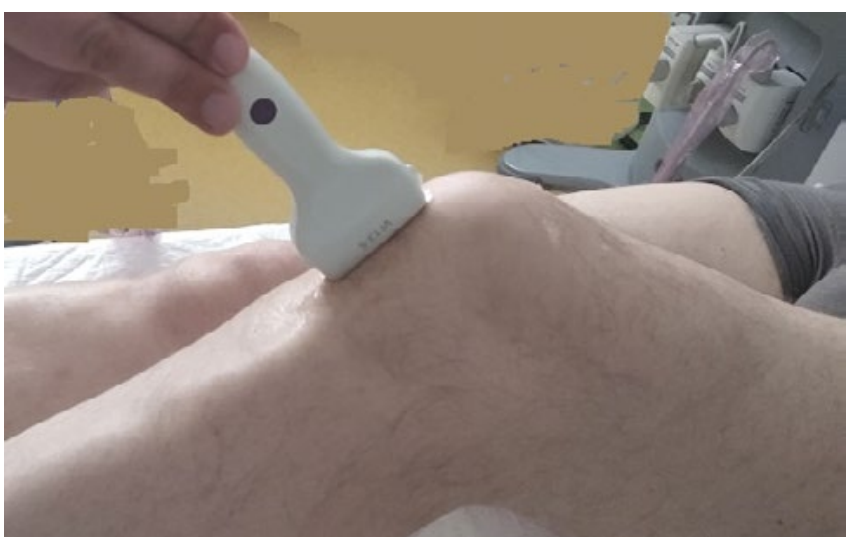

Fig. 13. Evaluation of the prepatellar bursa. Positioning of the patient and the transducer
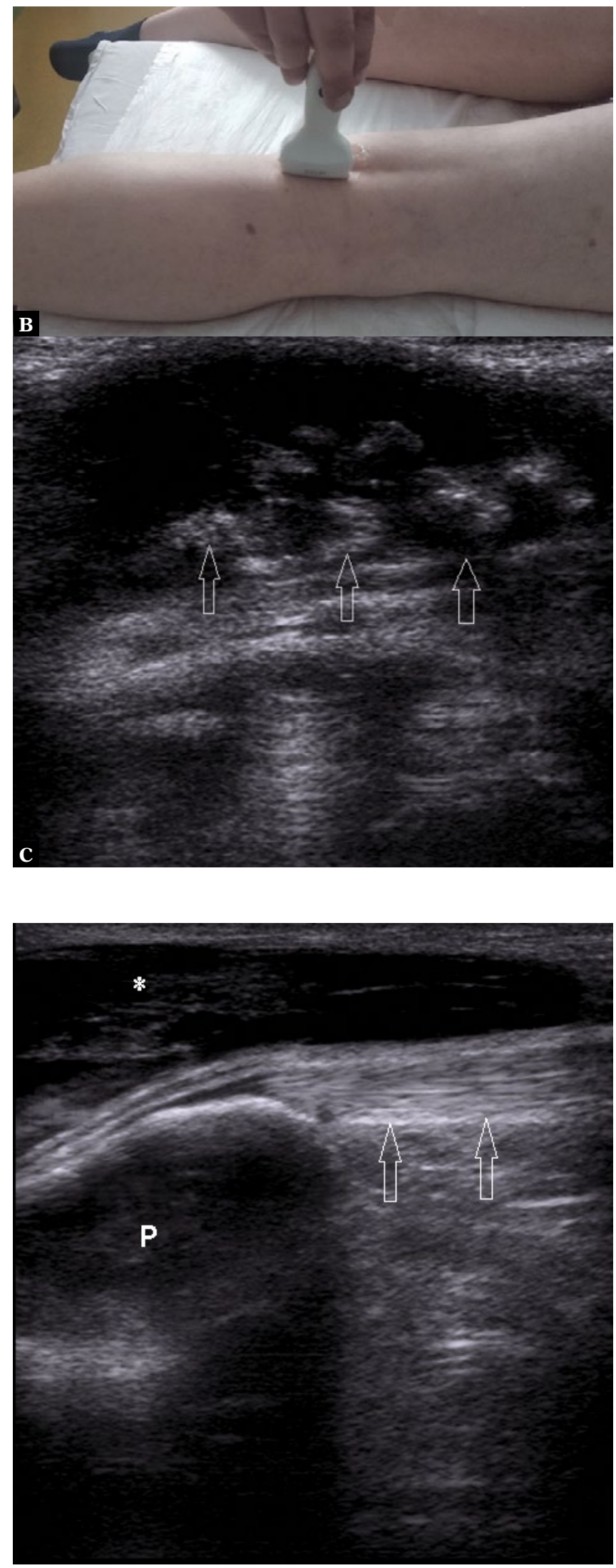

Fig. 14. Prepatellar bursitis (*). The prepatellar bursa is enlarged, filled with a copious amount of anechoic fluid and debris. P-patella. Arrows-patellar ligament 


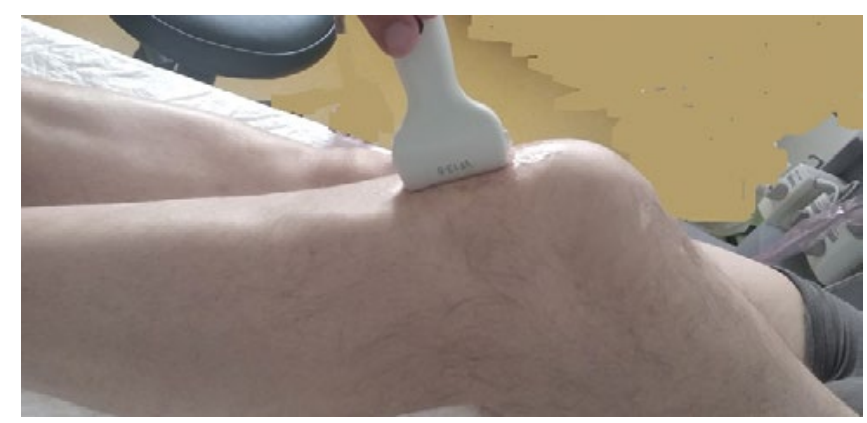

Fig. 15. Evaluation of the infrapatellar bursae. Positioning of the patient and the transducer

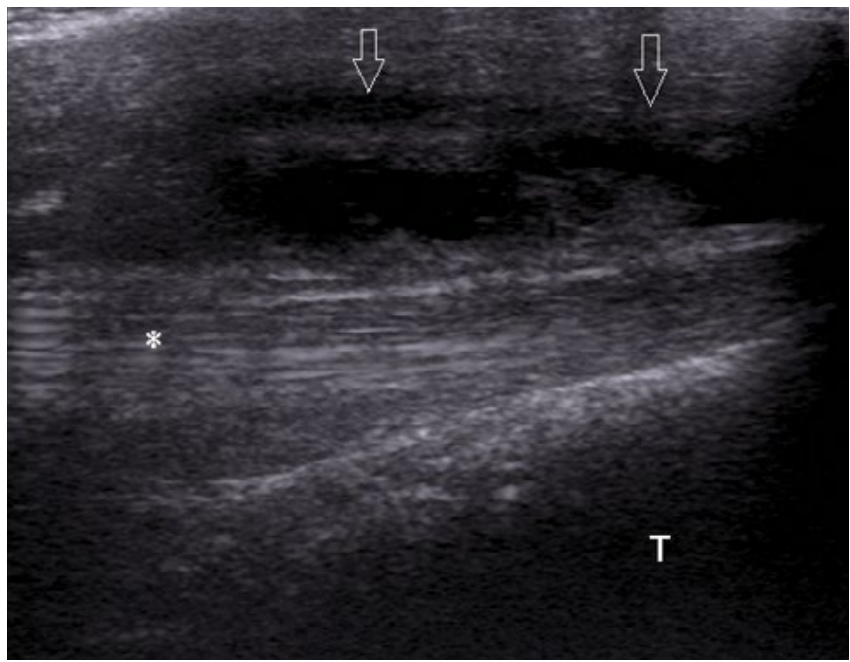

Fig. 16. Superficial infrapatellar bursitis (arrows). There is a moderate amount of fluid accumulated superficially to the distal part of the patellar ligament $\left(^{*}\right)$

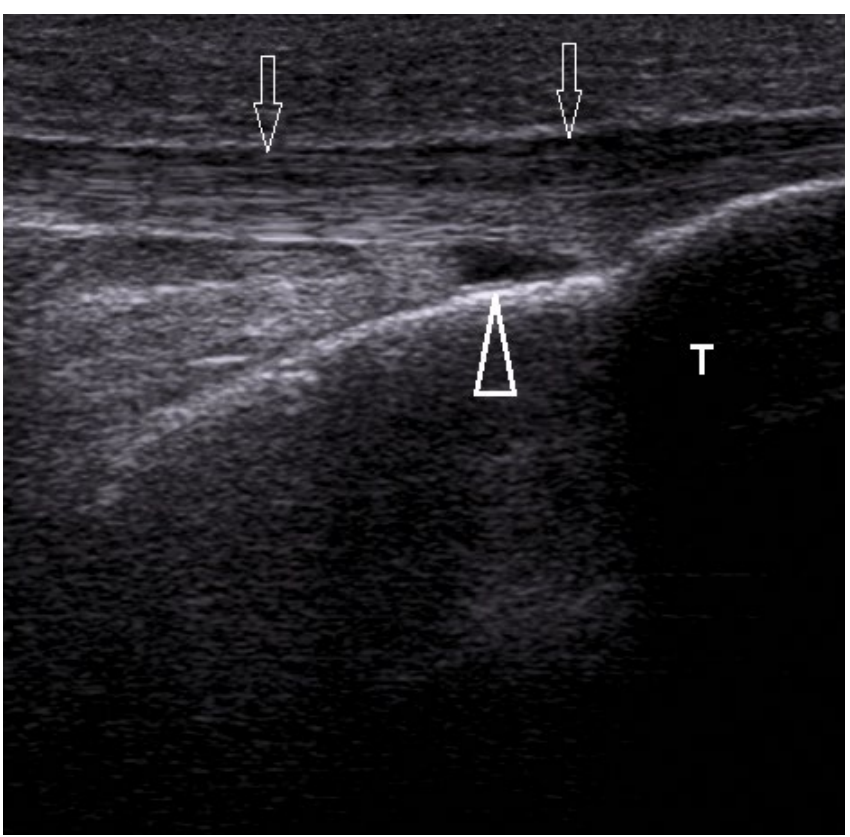

Fig. 17. Normal deep infrapatellar bursa can be seen as a small, triangular fluid collection (arrowhead) deep to the distal part of the patellar ligament (arrows), superficial to the tibial surface $(T)$
In order to evaluate both bursae by ultrasound, the patient should lie prone, with feet hanging over the bed. The calcaneal tuberosity can be used as bony landmark for identifying the bursae. The transducer should be positioned in the longitudinal plane, following the Achilles tendon (Fig. 19).

The deep retrocalcaneal bursa is more commonly affected by various inflammatory diseases, traumatic and overuse injuries than the superficial Achilles bursa. Inflammation of the deep retrocalcaneal bursa combined with insertional Achilles tendinopathy, formerly known as Haglund "impingement" syndrome ${ }^{(29,30)}$, is a common type of bursitis (Fig. 20). The superficial retrocalcaneal bursa is an adventitial bursa that can form after birth as a result of chronic friction, usually in people wearing tight shoes ${ }^{(29)}$.

\section{Conclusion}

High-resolution ultrasonography has many advantages in the imaging of the musculoskeletal system, including the imaging of the bursae, compared to other imaging methods, particularly in superficial, easily accessible parts of the body. The main advantages of the technique include ease of use, good acceptance by the patients, low cost of the examination in comparison to other modalities such as MRI, and the possibility of side-to-side comparison. It is arguably a perfect method for diagnosing the most common pathologies of the bursae. However, good knowledge of normal ultrasound anatomy of the bursae, their

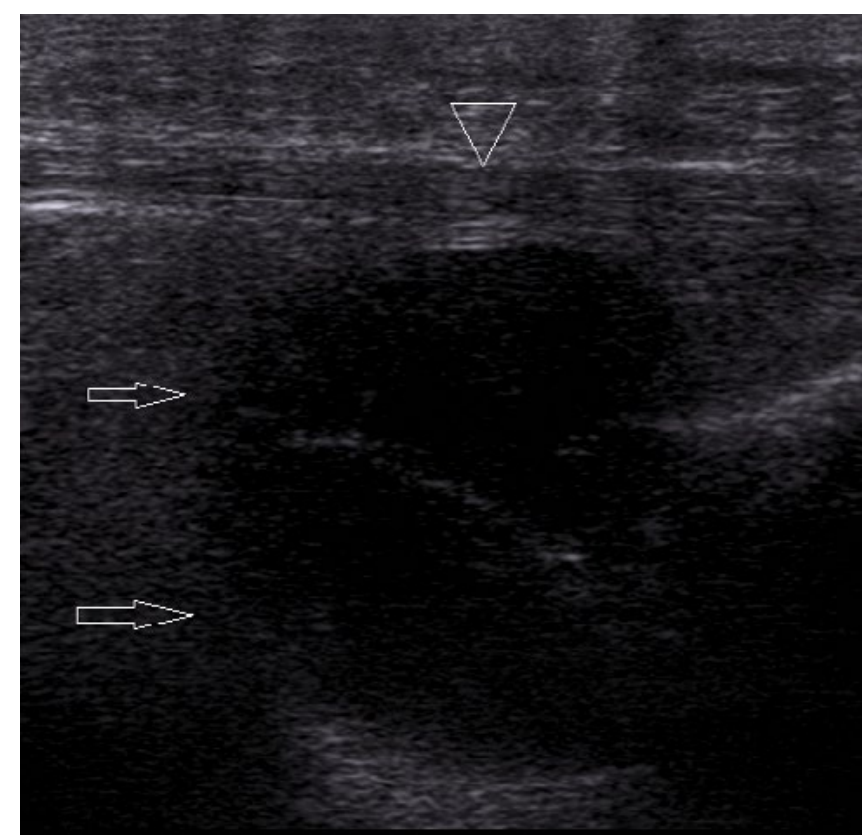

Fig. 18. Deep infrapatellar bursitis. A lobulated, enlarged deep infrapatellar bursa (arrows) deep to the distal part of the patellar ligament (arrowhead) 


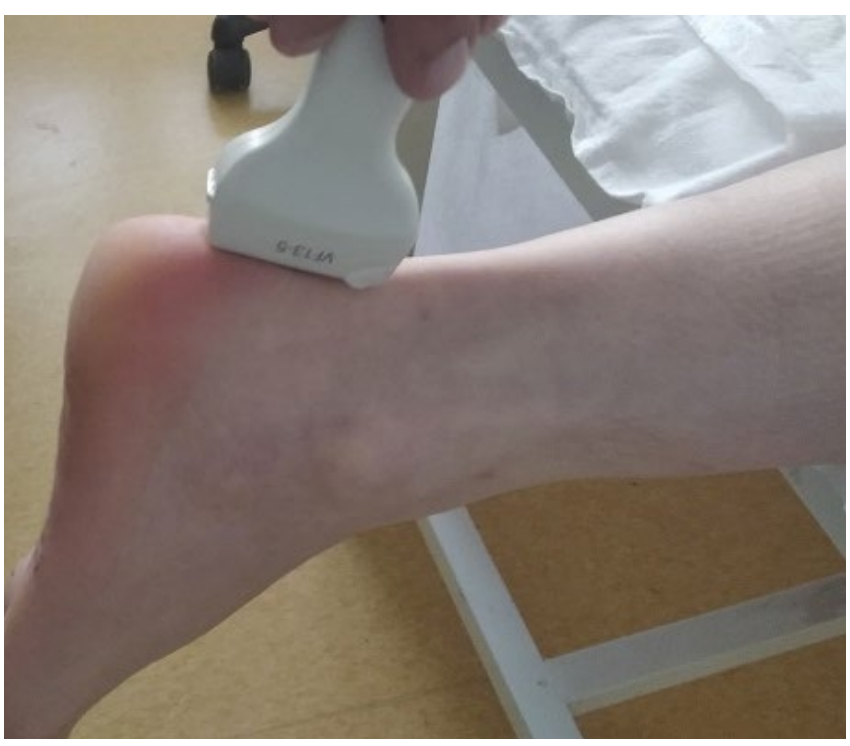

Fig. 19. Evaluation of the superficial and deep retrocalcaneal bursae. Positioning of the patient and the transducer

exact location and the most common bursal pathologies is essential for establishing quick and accurate diagnosis by ultrasound.

\section{References}

1. Hirji Z, Hunjun JS, Choudur HN: Imaging of the bursae. J Clin Imaging Sci 2011; 1: 22.

2. Lohr KM: Bursitis. Medscape Drugs and Diseases. New York, NY 2016. Available from: http://emedicine.medscape.com/article/2145588-overview.

3. DeLee JC, Drez D: Imaging effusions, cysts, and ganglia. In: DeLee JC, Drez D, Miller MD (eds): DeLee and Drez's Orthopaedic Sports Medicine: Principles and Practice. WB Saunders, Philadelphia 2003: 1646-1648.

4. Ruangchaijatuporn T, Gaetke-Udager K, Jacobson JA, Yablon CM, Morag Y: Ultrasound evaluation of bursae: anatomy and pathological appearances. Skeletal Radiol 2017; 46: 445-462.

5. Draghi F, Corti R, Urciuoli L, Alessandrino F, Rotondo A: Knee bursitis: a sonographic evaluation. J Ultrasound 2015; 18: 251-257.

6. Drakonaki EE, Allen GM, Wilson DJ: Ultrasound elastography for musculoskeletal applications. Br J Radiol 2012; 85: 1435-1445.

7. Kennedy MS, Nicholson HD, Woodley SJ: Clinical anatomy of the subacromial and related shoulder bursae: A review of the literature. Clin Anat 2017; 30: 213-226.

8. Martinoli C, Bianchi S, Prato N, Pugliese F, Zamorani MP, Valle M et al.: US of the shoulder: non-rotator cuff disorders. Radiographics 2003; 23: 381-401.

9. Meraj S, Bencardino JT, Steinbach L: Imaging of cysts and bursae about the shoulder. Semin Musculoskelet Radiol 2014; 18: 436-447.

10. van Holsbeeck MT, Strouse PJ: Sonography of the shoulder: evaluation of the subacromial-subdeltoid bursa. ARJ Am J Roentgenol 1993; 160: $561-564$.

11. Floemer F, Morrison WB, Bongartz G, Ledermann HP: MRI characteristics of olecranon bursitis. AJR Am J Roentgenol 2004; 183: 29-34.

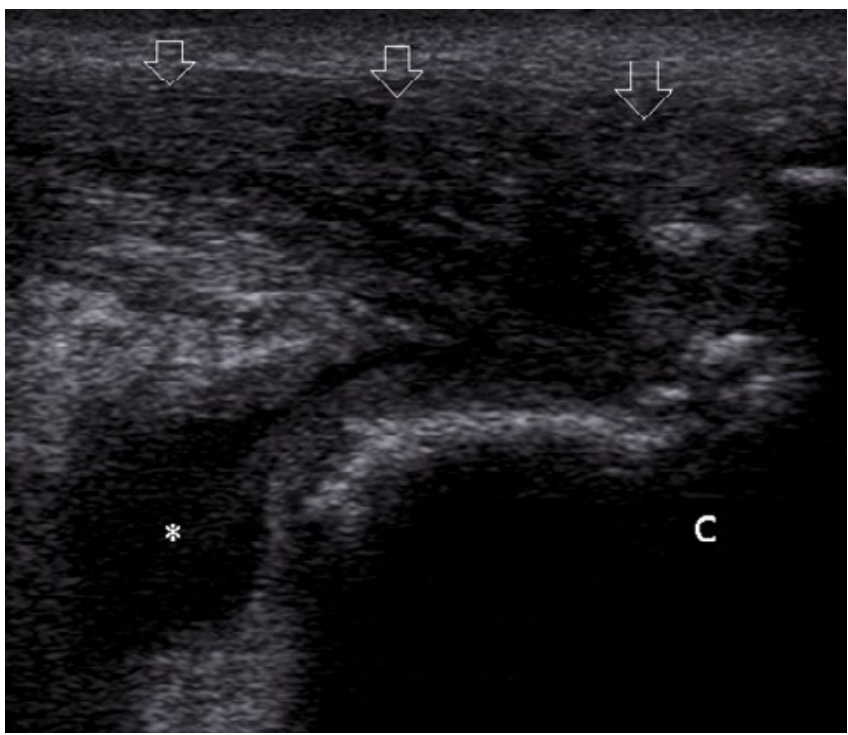

Fig. 20. Deep retrocalcaneal bursitis (*) in a patient with insertional Achilles tendinopathy (arrows). The Achilles tendon is swollen, with disrupted fibrillar pattern and calcifications near the insertion. $C$-calcaneus

\section{Conflict of interest}

The authors do not report any financial or personal connections with other persons or organizations which might negatively affect the contents of this publication and/or claim authorship rights to this publication.

12. Blackwell JR, Hay BA, Bolt AM, Hay SM: Olecranon bursitis: a system atic overview. Shoulder Elbow 2014; 6: 182-190.

13. Pfirrmann CW, Chung CB, Theumann NH, Trudell DJ, Resnick D: Greater trochanter of the hip: attachment of the abductor mechanism and a complex of three bursae - MR imaging and MR bursography in cadavers and MR imaging in asymptomatic volunteers. Radiology 2001; 221: 469-477.

14. Long SS, Surrey DE, Nazarian LN: Sonography of greater trochanteric pain syndrome and the rarity of primary bursitis. AJR Am J Roentgenol 2013; 201: 1083-1086.

15. Varma DG, Richli RW, Charnsangavej JC, Samuels BI, Kim EE, Wallace S: MR appearance of the distended iliopsoas bursa. AJR Am J Roentgenol 1991; 156: 1025-1028.

16. Lin YT, Wang TG: Ultrasonographic examination of the adult hip. J Med Ultrasound 2012; 20: 201-209.

17. Skiadas V, Koutoulidis V, Plotas A: An atypical case of noninfected iliopsoas bursitis - MRI findings. J Radiol Case Rep 2009; 3: 15-18.

18. Steinbach LS, Stevens KJ: Imaging of cysts and bursae about the knee. Radiol Clin North Am 2013; 51: 433-454.

19. Ostergaard M, Court-Payen M, Gideon P, Wieslander S, Cortsen M, Lorenzen I et al.: Ultrasonography in arthritis of the knee: A comparison with MR imaging. Acta Radiol 1995; 36: 19-26.

20. Karim Z, Wakefield RJ, Quinn M, Conaghan PG, Brown AK, Veale DJ et al.: Validation and reproducibility of ultrasonography in the detection of synovitis in the knee: A comparison with arthroscopy and clinical examination. Arthritis Rheum 2004; 50: 387-394.

21. Alves TI, Girish G, Kalume Brigido M, Jacobson JA: US of the knee: scanning techniques, pitfalls, and pathologic conditions. Radiographics 2016; 36: 1759-1775. 
22. Draghi F, Urciuoli L, Alessandrino F, Corti R, Scudeller L, Grassi R: Joint effusion of the knee: potentialities and limitations of ultrasonography. J Ultrasound 2015; 18: 361-371.

23. Telischak NA, Wu JS, Eisenberg RL: Cysts and cystic-appearing lesions of the knee: A pictorial essay. Indian J Radiol Imaging 2014; 24 : 182-191.

24. Chhabra A, Cerniglia CA: Bursae, cysts and cyst-like lesions about the knee. J Am Osteopath Coll Radiol 2013; 2: 2-13.

25. McCarthy CL, McNally EG: The MRI appearance of cystic lesions around the knee. Skeletal Radiol 2004; 33: 187-209.

26. Perdikakis E, Skiadas V: MRI characteristics of cysts and "cyst-like" lesions in and around the knee: What the radiologist needs to know. Insights Imaging 2013; 4: 257-272.
27. Schmidt WA, Schmidt H, Schicke B, Gromnica-Ihle E: Standard reference values for musculoskeletal ultrasonography. Ann Rheum Dis 2004; 63: 988-994.

28. Mathieson JR, Connell DG, Cooperberg PL, Lloyd-Smith DR: Sonography of the Achilles tendon and adjacent bursae. AJR Am J Roentgenol 1988; 151: 127-131.

29. van Dijk CN, van Sterkenburg MN, Wiegerinck JI, Karlsson J, Maffulli N: Terminology for Achilles tendon related disorders. Knee Surg Sports Traumatol Arthrosc 2011; 19: 835-841.

30. Sella EJ, Caminear DS, McLarney EA: Haglund's syndrome. J Foot Ankle Surg 1998; 37: 110-114. 